\title{
Commonplace
}

\section{Curating a Public Conversation about Annotation}

\author{
Remi Kalir1 ${ }^{1}$, Antero Garcia ${ }^{2}$ \\ ${ }^{1}$ University of Colorado Denver, ${ }^{2}$ Stanford University
}

Published on: Jul 22, 2021

License: Creative Commons Attribution 4.0 International License (CC-BY 4.0). 
Dear Reader,

Writing a book about annotation, titled Annotation, we embraced the inevitable: Readers would write in our book.

Some readers' marginalia in Annotation might remain private. Perhaps that was - or will be- your choice as a reader and writer. Then again, maybe your comments on Annotation are circulating across social media. Maybe you also participated in the open peer review of our book, or perhaps you've added a public rating or review about Annotation to various websites. You, our reader, with your notes tethered to Annotation, have made visible and extended the social life of annotation.

In presenting the ubiquity and importance of notes added to texts, we knew it would be necessary - and also illustrative - to amplify the messy, layered, and colloquially "meta" qualities of annotation as an everyday literacy practice.

Reading has been a social and publicly shared practice for centuries. Today, contemporary social media has amplified the expressive register of reader response. You've likely shared, or certainly seen, pictures of highlighted and marked-up books on Twitter and Instagram. Book titles become hashtags. Facebook posts can function as de facto book reviews that elicit subsequent emoji-based reactions from your friends. Considering all of this, we anticipated that your reading of, writing in, and commentary about Annotation would be shared with others and perhaps also made public, and so we sought to guide some aspect of your response.

As described in our complementary Commonplace reading list, annotation thrives beyond the printed page as readers write on and about the built environment, interact with digital media, and make shared meaning across their everyday texts and contexts. For academics, annotation is also essential to scholarly communication and knowledge production. With Annotation, we eagerly accepted a social and scholarly responsibility to spark, curate, and facilitate discussion about annotation.

So as to help name and also sustain this conversation about annotation, we created \#AnnoConvo, an abbreviation, a hashtag, and an invitation to share. Perhaps you've already read Annotation and participated in our \#AnnoConvo, or maybe you'll do so now.

This post - which we've written as a brief dialogue guided by examples - describes the origin of \#AnnoConvo, features some recent contributions to this public conversation, 
and also addresses tensions associated with marks in books and, more broadly, the power of annotation.

Remi Kalir: Antero, let's revisit the open peer review of Annotation from the summer of 2019. That was, in many respects, the beginning of our public annotation conversation with readers. What remains remarkable to you, and what was valuable about that process?

Antero Garcia: Honestly, I think the part that sticks with me is how much of a dialogue that process felt like! We had more than two dozen deeply engaged readers thinking about and adding to our work. It was so much more than a traditional peer review process and we were able to carry forward the generosity of public annotation into the final version of the book, and, in some ways, into the curation of \#AnnoConvo itself. Four hundred ninety-one annotations totaling 20,275 words!

RK: And, because of that, our book contains these custom illustrations that feature direct quotes from the open peer review, some of those digital annotations became printed commentary.

AG: These pages in Annotation have key ideas and quotes from our writing process that we encourage readers now to respond to, and whether handwritten in ink, or by tweet, or by whatever modality might feel right in the moment! 
"Annotation seems to be something, while

done with intention, that happens in the

moment. It is fleeting, though meaningful."

Annotation in the strict sense in no way differs from intertextuality. It is intertextual through and through, from the moment we understand "text" in the classical sense as a notation representing one discourse propped on another. It consists, in effect, of a text related to another text that has meaning only within the relationship. - Derrida

"This is so critical - the (externalized) dialogic nature of annotation. Not only am I interacting with and responding to text internally, I am engaging in the dialogue externally."

Figure 5

(L) Annotation Figure 5 on page 24 features two comments from the PubPub open peer review, and (R) Annotation Figure 5 annotated with handwritten comment by_ Paulo Pinto(@w3bk3rn3l)_and shared via Twitter.

RK: Like Paulo's handwritten annotation about intertextuality that mimics in style the printed - and previously digital- annotations from open peer review.

AG: Looking at the tactility of writing and highlighting on a physical page and then also tweeting an image of that process, Paulo's creation reminds us of the lived historicity that Davis and Mueller (2020) describe: "The page is now a place that enables forms of textual activity both new and old: clicking, scrolling, reading, embedding, interacting, commenting, annotating."

RK: There's a playful quality to this type of reader response, don't you think?

AG: Yeah, this interaction can look a lot like the playfulness of what Kevin Hodgson shares in his \#AnnoConvo tweet below, and in the many tweets he authored as one of the earliest readers of the final, published book! Remi, we talk specifically about the kinds of blackout poetry that Kevin's tweet utilizes in the book. I'm curious if you had 
thoughts on the kinds of annotation that Kevin is doing here and what it might mean for readers?

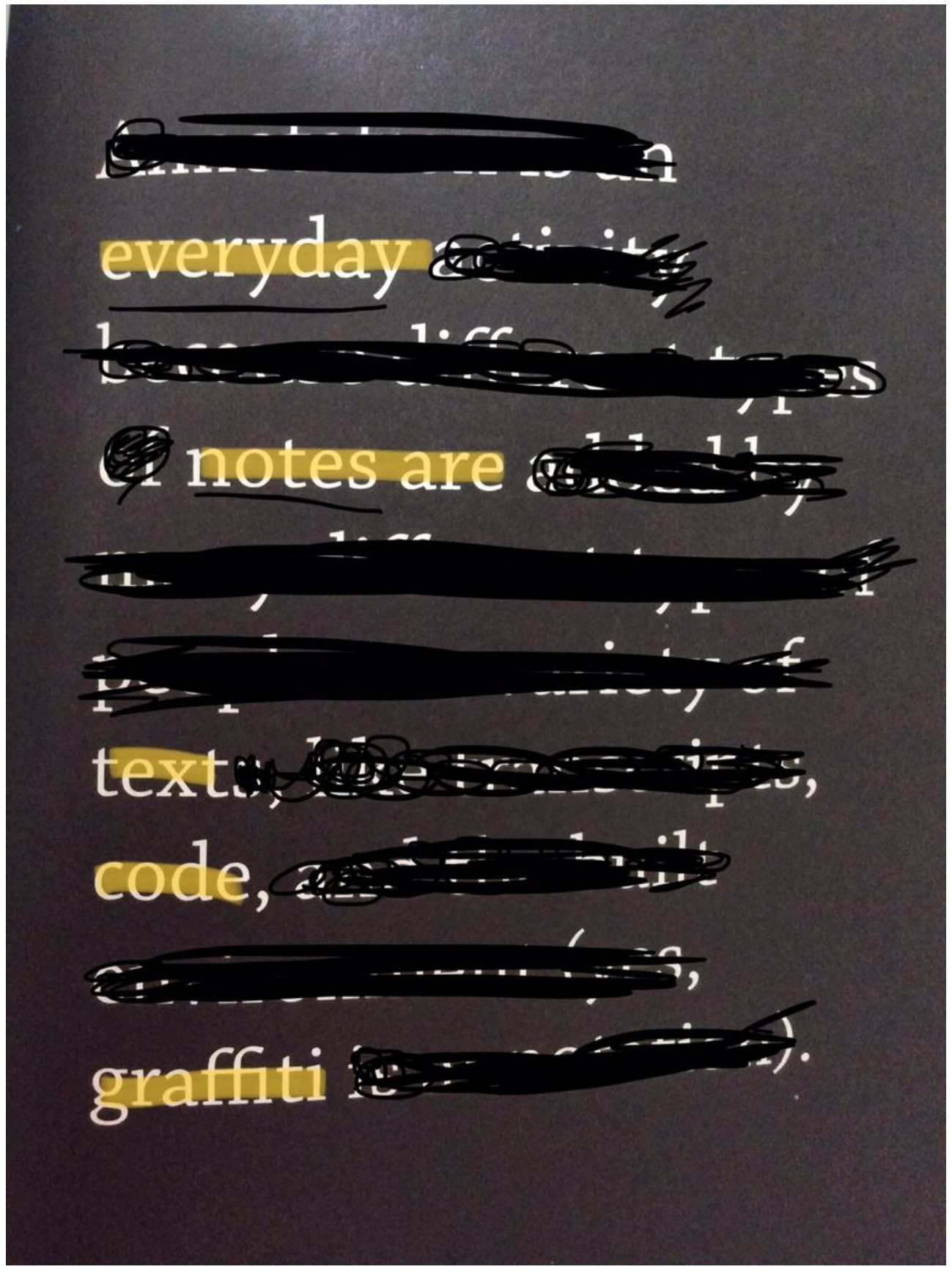

A digital "poem" created by Kevin Hodgson (@dogtrax)_and shared via Twitter. 
RK: Let's unpack Kevin's contribution to \#AnnoConvo. First, Kevin took a photograph of our book, a quote that appears on page 11, presumably with a phone. Then, Kevin drew, literally and figuratively, on the conventions of blackout poetry, and added redaction - a form of annotation - atop this digital photograph of the printed page. Six words are also digitally highlighted, yet another form of annotation. This composite image was then shared via Twitter using two hashtags (more annotation!), both \#AnnoConvo and also \#smallpoems, with the added note: "removal of text is another layer of interaction with the text ..." The social life of any text, like Annotation, ebbs and flows, awaiting the playful and provocative engagement from readers like Kevin.

AG: But engaging with our book doesn't necessarily mean writing in the book.

RK: Indeed, and that brings us to the next tweet.

Victor R. Lee

@VicariousLee

I really respect that @remikalir and @anterobot have a book out on Annotation and like that people post how much they like annotating it. However, it makes me uncomfortable having grown up owning few books and feeling very afraid to damage them by writing in them.

9:43 PM · Jun 3, 2021 - Twitter for iPhone

\section{Retweets $\mathbf{2}$ Quote Tweets $\mathbf{2 1}$ Likes}

Tweet by Victor Lee about Annotation.

RK: Let's conclude with this example from your colleague, Victor Lee. We began a recent talk about Annotation with Victor's tweet. His perspective on access, ownership, and power helped us to discuss a tension between readers who can and do write annotation -whether in books or the built environment- and the cultural rites of annotation, often unwritten, that also constrain where and how notes are added to 
everyday texts. Revisiting Victor's tweet, in light of this post about \#AnnoConvo, how might readers better understand the complexities of writing annotations and, more specifically, their participation in an annotated conversation about our book

AG: Honestly, I'm really grateful for the ways Victor's tweet opens up the conversation around annotation. We spent a lot of time talking about power in Annotation.

RK: We do, even asking in our conclusion, "How might the social life of annotation serve the public good?" Any social benefit mediated by annotation must address power.

AG: Victor helps remind us of the ways that power -and who gets to annotate- is bound by social norms, cultural practices, and enforced policies. It's not enough to just encourage people to engage in the playfulness that Kevin or Paulo's tweets demonstrate. Instead, we need to think about how annotation practices-and the possibilities for democratic participation-must be cultivated and encouraged.

RK: As readers chose to write in Annotation, and perhaps also share their thoughts via \#AnnoConvo, I do hope the creative attributes of annotation are accompanied by critical expressions and assessments, too. It's humbling, and exciting, to know that our book can help advance a more nuanced dialogue about what prevents and also permits readers from writing in their books-and, by extension, in writing about their world. 